MATHEMATICS OF COMPUTATION

Volume 66, Number 218, April 1997, Pages 477-493

S 0025-5718(97)00825-9

\title{
A NUMERICAL SCHEME BASED ON MEAN VALUE SOLUTIONS FOR THE HELMHOLTZ EQUATION ON TRIANGULAR GRIDS
}

\author{
M. G. ANDRADE AND J. B. R. DO VAL
}

\begin{abstract}
A numerical treatment for the Dirichlet boundary value problem on regular triangular grids for homogeneous Helmholtz equations is presented, which also applies to the convection-diffusion problems. The main characteristic of the method is that an accuracy estimate is provided in analytical form with a better evaluation than that obtained with the usual finite difference method. Besides, this classical method can be seen as a truncated series approximation to the proposed method. The method is developed from the analytical solutions for the Dirichlet problem on a ball together with an error evaluation of an integral on the corresponding circle, yielding $O\left(h^{4}\right)$ accuracy. Some numerical examples are discussed and the results are compared with other methods, with a consistent advantage to the solution obtained here.
\end{abstract}

\section{INTRODUCTION}

In this paper we consider numerical solutions on triangular mesh grids for the Helmholtz equation $\Delta u-\lambda^{2} u=0$, with the use of a method that we should call henceforth the Mean Value Scheme (MVS). The method is based on the same central ideas of the method in [14] for square mesh grids; here, it is adapted for the triangular mesh grids, with an improvement in the accuracy. The point of view adopted is that of an approximation for the integral on the circle, associated to the solution for the Helmholtz equation on the interior of this special domain, instead of the usual discretization of the differential operator. This type of analytical result can be seen as an extension to the Helmholtz equation, of the mean value theorem for harmonic functions, which explain the allusion in the given name. The proposed method can also be applied in a straightforward manner to the convection-diffusion equations $-\Delta u+\alpha \cdot \nabla u+q u=0$, with $\alpha$ a constant vector in $\mathbb{R}^{2}$ and $q$ a nonnegative scalar.

In the present setting, we consider circles that are centered on each mesh point and contain the six neighboring points in the triangular mesh grid; a partial covering

Received by the editor July 31, 1995.

1991 Mathematics Subject Classification. Primary 35A40, 65N06; Secondary 35J25, 65N15, $65 \mathrm{~N} 22$

Key words and phrases. Numerical solutions for partial differential equations, elliptic differential equations, Helmholtz equations, non-standard difference approximation, convection-diffusion equations.

This work was partially supported by CNPq, Conselho Nacional de Desenvolvimento Científico e Tecnológico, grant number 300573/95-2(NV) and 300721/86-2(NV).

(C) 1997 American Mathematical Society 
for the domain composed of an intertwined set of balls is defined by this construction. The six points on the circle provide an approximation to a line integral on the circle associated with the "mean value" calculus for the Helmholtz equation. An improvement in the precision is obtained here, when compared with the evaluation presented in [14], since the number of points employed increased from four to six.

The standard finite difference method for Helmholtz equations with triangular mesh grids can be traced from [10], and we shall refer to it as the Finite Difference Scheme (FDS). In an analytical comparison presented in section 3, it is shown that the FDS in fact stands as an approximation to the MVS. This conclusion is straightforward since the modified Bessel function of zero order, which arises in the proposed method, possesses the first three terms in its series representation identical to the three terms appearing in the standard FDS. The precision error for the MVS and the FDS is of order $O\left(h^{4}\right)$ ( $h$ is the mesh grid step size), however, we can show that a tighter upper bound for the precision error holds for the MVS.

The precision error of the proposed method is compared numerically with other difference schemes: the Single Cell High Order Scheme (SCHOS), defined on a single square cell of size $2 h$ over a 9-point stencil, according to [11]; the Discrete Weighted Mean Approximation (DWMA), developed in [4] for a specific class of problems and the Quadratic Influence Scheme (QIS), defined for a 9-point stencil, as in [13]. Notice that the MVS is a 7-point scheme which naturally yields simpler matrices than that obtained in a 9-point scheme, thus requiring less effort. The largest precision that can be attained in regular grids with a 9-point scheme for the Helmholtz equation is $O\left(h^{6}\right)$, see [11] and [1], which lead us to believe that the precision error of order $O\left(h^{4}\right)$ is probably the precision error limit for the equation studied in triangular mesh grids. In [1], the authors concentrate on finite difference schemes for the Helmholtz equation, and they remark that they can see no natural way to derive any equally good 9-point stencil from variational principles, which somehow explains the permanence of finite difference methods even with a plethora of finite element methods. The comparisons of the MVS with the three abovementioned methods are presented in section 6 for some examples studied in [8], [9] and [4] with a consistent advantage to the MVS.

It is mentioned in different contexts that when the product $\lambda \times h$ is large the methods generally provide poor results, e.g. [8], [9], [4], [13]. We also verify here this general behavior for the FDS and the MVS in some numerical experiments, but the results show that the MVS presents a good precision in a range of $\lambda \times h$ that is not attained by any of the methods known by the authors, see section 6 . This suggests a wide application of the MVS to the so-called convection-diffusion problem.

The paper is organized as follows. In section 2 the preliminary definitions are given and in section 3 the main result (Theorem 3.1 and Corollary 3.1) is presented. An analytical comparison with the FDS is developed and the advantage of the MVS method is inferred from a favorable comparison between the precision error upper bound for both methods. In section 4 the arguments in preparation for the proofs are devised: the covering of the given domain with a set of two-dimensional balls, and the error evaluation for the numerical approximation for the integral arising from the mean value theorem for the Helmholtz equation. In Section 5 the proof of the main results is developed from the previous results and from the construction of the matrices involved. Section 6 presents numerical examples and comparisons that underline the improvement in the accuracy obtained by the MVS method. 
Analytical solutions are employed for the comparisons among the MVS, the FDS, the SCHOS and the DWMA methods, and the maximum relative and absolute errors are exhibited. Some numerical estimates for the order of the discretization errors are also presented.

\section{Problem formulation And Basic Definitions}

Let $\Omega$ be an open and limited subset of the Euclidean two-dimensional space, with boundary denoted by $\partial \Omega$ and $\bar{\Omega}=\Omega \cup \partial \Omega$, the closure of $\Omega$. The pair $(x, y)$ will denote a point in $\bar{\Omega}$. Let $u: \bar{\Omega} \mapsto \mathbb{R}$ be a real function defined on $\bar{\Omega}$, and denote by $\mathbb{C}^{0}(\Omega)$ (or $\mathbb{C}^{0}(\partial \Omega)$ ) the class of bounded and continuous functions on $\Omega$ (or $\partial \Omega$ ) and by $\mathbb{C}^{k}(\Omega)$ the class of functions that is continuous and possesses continuous partial derivatives in $\Omega$ of order $k$. Let us consider a function $u(x, y) \in \mathbb{C}^{6}(\Omega) \cap \mathbb{C}^{0}(\bar{\Omega})$ satisfying the following standard Dirichlet problem:

$$
\text { Problem }(\Sigma): \begin{cases}\Delta u(x, y)-\lambda^{2} u(x, y)=0, & (x, y) \in \Omega \\ u(x, y)=\Psi(x, y), & (x, y) \in \partial \Omega .\end{cases}
$$

We assume that the basic conditions for the existence of a unique solution is satisfied, e.g., [5].

In order to solve the problem numerically for a general domain $\Omega$, it is necessary to introduce a discrete grid on $\bar{\Omega}$ and evaluate $u$ approximately at each point (node). We adopt the uniform triangular grid as follows.

Definition 2.1. Let $G_{h}$ denote a uniform triangular grid with step $h$ covering the region $\bar{\Omega}$ such that for some $N$ and $M$

$$
\begin{aligned}
G_{h}:=\left\{\left(x_{i}, y_{j}\right) \in \bar{\Omega}, i=0\right. & , 1,2, \cdots, N, j=0,1,2, \cdots, M \\
& \left.\mid x_{i}=x_{0}+i h+p h \cos (\pi / 3), y_{j}=y_{0}+j h \sin (\pi / 3)\right\},
\end{aligned}
$$

where $p:=(j \bmod 2)-1$. A node in this grid is denoted by $z_{i, j}=\left(x_{i}, y_{j}\right)$, and we can associate to any function $\phi: \bar{\Omega} \mapsto \mathbb{R}$ a discrete function $\widehat{\phi}: S_{h} \mapsto \mathbb{R}$ called grid function, such that $\widehat{\phi}_{i, j}=\phi\left(z_{i, j}\right)$. A node $z_{i, j}$ of $G_{h}$ is internal if the distance from $z_{i, j}$ to $\partial \Omega$ is larger than $h \sqrt{3} / 2$ in the $y$-coordinate direction and larger than $h$ in the $x$-coordinate direction. The total number of interior points is denoted by $N_{0} M_{0}$ with $N_{0}=N-1$ and $M_{0}=M-1$.

For $v \in \mathbb{R}^{n}$ and $A \in \mathbb{R}^{n \times n}, v_{i}$ and $a_{i, j}$ denote respectively a generic element of $v$ and $A$. We adopt in what follows the usual sup norm for $v$ and $A$ [6]. For a function $u \in \mathbb{C}^{k}(\Omega)$ let $D_{k}$ denote an upper bound for the $k$-th order differentials of $u$, namely

$$
D_{k}:=\max \left\{\sup \left\{\left|\frac{\partial^{k} u(x, y)}{\partial x^{k-j} y^{j}}\right|:(x, y) \in \Omega\right\}: 0 \leq j \leq k\right\} .
$$

Also, we denote by $I_{0}: \mathbb{R} \mapsto \mathbb{R}_{+}$the modified Bessel function of zero order, represented by the series

$$
I_{0}(x)=\sum_{k=0}^{\infty} \frac{x^{2 k}}{2^{2 k}(k !)^{2}} ; \quad x \in \mathbb{R} .
$$




\section{MAin RESUlts}

Theorem 3.1. Let $u(x, y) \in \mathbb{C}^{6}(\Omega)$, be the solution for the boundary value problem $\Sigma$, and let $G_{h}$ be the triangular grid, given in Definition 2.1, and $u_{i, j}$ be the correspondent grid function. Let $\left\{U_{i, j}\right\}$ be a grid function that satisfies the following difference equation for $i=0,1,2, \cdots, N_{0}, j=0,1,2, \cdots, M_{0}$,

$$
U_{i, j}=\frac{1}{6 I_{0}(\lambda h)}\left(U_{i-1, j-1}+U_{i+1, j-1}+U_{i-2, j}+U_{i+2, j}+U_{i-1, j+1}+U_{i+1, j+1}\right) .
$$

It follows that

$$
\|u-U\|_{\infty} \leq \frac{1}{I_{0}(\lambda h)-1}\left(\frac{h^{6} D_{6}}{6 !}+O\left(h^{7}\right)\right) .
$$

Corollary 3.1. Let $u(x, y) \in \mathbb{C}^{6}(\Omega)$ be the solution for the boundary value problem $\Sigma$, and $G_{h}$ be the triangular grid, according to Definition 2.1. If $U=\left\{U_{i, j}\right\}$ is a grid function satisfying (3.1), then

$$
\|u-U\|_{\infty}=O\left(h^{4}\right) .
$$

Remark 3.1. The difference equation in Theorem 1 can be compared to the standard difference equation obtained for the Helmholtz equation when the grid $G_{h}$ is adopted, e.g. [10]. Using the Taylor series, equation (3.1) has a standard similar counterpart given by

$$
U_{i, j}=\frac{1}{6 T_{1}(\lambda h)}\left(U_{i-1, j-1}+U_{i+1, j-1}+U_{i-2, j}+U_{i+2, j}+U_{i-1, j+1}+U_{i+1, j+1}\right),
$$

where

$$
T_{1}(\lambda h)=1+\frac{(\lambda h)^{2}}{4}+\frac{(\lambda h)^{4}}{64} .
$$

The truncation error for (3.4) is obtained by direct development of the Taylor series for $u(x, y)$ in a point in $G_{h}$, and by substituting the basic relation $\Delta u=\lambda^{2} u$ and $\Delta^{2} u=\Delta(\Delta u)=\lambda^{4} u$ into the sum of the six series involving the six neighboring points. Denoting by $\tau\left\{u_{i, j}\right\}$ the truncation error, we obtain

$\tau\left\{u_{i, j}\right\}=u_{i-1, j-1}+u_{i+1, j-1}+u_{i-2, j}+u_{i+2, j}+u_{i-1, j+1}+u_{i+1, j+1}-6 T_{1}(\lambda h) u_{i, j}$

and the analysis in [10] yields the following bound, which is justified for a function $u \in \mathbb{C}^{6}(\Omega)$ :

$$
\begin{aligned}
\tau\left\{u_{i, j}\right\} & =\frac{6 h^{6}}{16 \times 6 !}\left(33 \frac{\partial^{6} u_{i, j}}{\partial x_{i}^{6}}+45 \frac{\partial^{6} u_{i, j}}{\partial x_{i}^{4} \partial y_{j}^{2}}+135 \frac{\partial^{6} u_{i, j}}{\partial x_{i}^{2} \partial y_{j}^{4}}+27 \frac{\partial^{6} u_{i, j}}{\partial y_{j}^{6}}\right)+O\left(h^{7}\right) \\
& \leq \frac{15 h^{6}}{6 !} D_{6}+O\left(h^{7}\right) .
\end{aligned}
$$

The precision error can now be evaluated using the following upper bound developed in this work, see Lemma 3.1 in the sequel 


$$
\|u-U\|_{\infty} \leq \frac{1}{6 T_{1}(\lambda h)-6}\|\tau\{u\}\|_{\infty}
$$

From (3.5), we now have that

$$
\|u-U\|_{\infty} \leq \frac{1}{6 T_{1}(\lambda h)-6}\left(\frac{15 h^{6}}{6 !} D_{6}+O\left(h^{8}\right)\right) .
$$

Lemma 3.1. Suppose that the finite difference schemes in (3.1) and (3.4) yield, respectively, the following matrix representation:

$$
A_{I_{0}} U=b, \quad A_{T_{1}} U=b
$$

with $b \in \mathbb{R}^{N_{0} M_{0}}$ and $A_{I_{0}}$ and $A_{T_{1}}$ of dimension $N_{0} M_{0} \times N_{0} M_{0}$. Then

$$
\left\|A_{I_{0}} v\right\|_{\infty} \geq 6\left(I_{0}(\lambda h)-1\right)\|v\|_{\infty}, \quad\left\|A_{T_{1}} v\right\|_{\infty} \geq 6\left(T_{1}(\lambda h)-1\right)\|v\|_{\infty}, \quad \forall v \in \mathbb{R}^{N_{0} M_{0}} .
$$

The proof of Lemma 3.1 is postponed to section 5 .

Remark 3.2. The basic distinction between expressions (3.1) and (3.4) is the modified Bessel function in the denominator in (3.1), and the polynomial $T_{1}(\lambda h)$ appearing in the denominator in (3.4). Distinct evaluations for the precision error are obtained by comparing (3.2) and (3.7), with an advantage for the MVS method, presented in Theorem 3.1. This can be verified first from a factor of $15 / 6$ in the right-hand side of (3.7); also notice that the denominator of (3.2) presents the infinite series $I_{0}(\lambda h)$ in place of the polynomial $T_{1}(\lambda h)$ arising in the standard finite difference method. In addition, the function $T_{1}(\lambda h)$ corresponds to the first three terms of the series represented by $I_{0}(\lambda h)$ which clearly shows the consistency of the two schemes, and demonstrates that the Taylor series development represents an approximation for the method proposed here. The ratio between the two coefficients appearing in (3.2) and (3.7) yields the following expression:

$$
R:=\left(\frac{15}{6 T_{1}(\lambda h)-6}\right)\left(\frac{1}{I_{0}(\lambda h)-1}\right)^{-1}=\frac{15}{6}\left[1+\frac{1}{T_{1}(\lambda h)-1}\left(\sum_{k=3}^{\infty} \frac{(\lambda h)^{2 k}}{2^{2 k}(k !)^{2}}\right)\right] .
$$

Fig. 1 shows a plot for ratio $R$, picturing the improvement in the error bound associated with the proposed method for different values of parameter $\lambda$ and grid size $h$. The justification behind the result in Theorem 3.1 relies on the solution of the Dirichlet problem defined locally on an intertwined set of balls covering the domain. This procedure leads to the solution of problem $\Sigma$ as assured by the Alternating Method of Schwarz, e.g. [2, chap. VII], [12], applied to the set of balls. The Dirichlet problem for the Helmholtz equation defined on the interior of one ball possesses an analytical form, see Lemma 4.1. A numerical approximation to the line integral on the circle is proposed and the numerical error is evaluated. These steps are developed in the next section. With these elements, the adequate scenario for the proof of Theorem 3.1 is settled, and section 5 is devoted to the proof of the main results. 


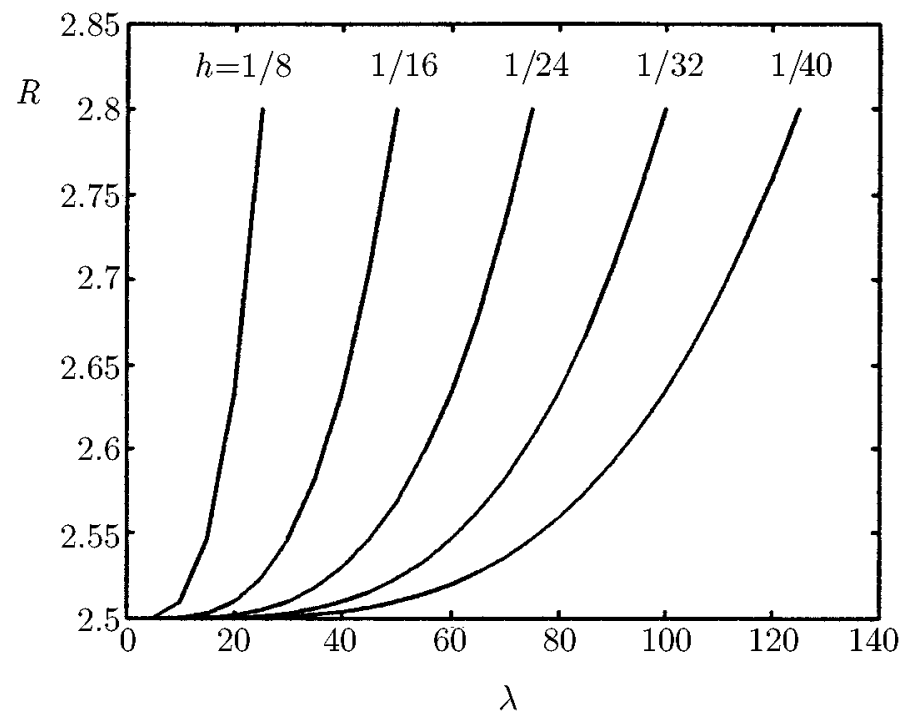

Figure 1. A plot for $\frac{15}{6} \frac{I_{0}(\lambda h)-1}{T_{1}(\lambda h)-1}$

\section{COVERING $\Omega$ With BALls AND NUMERICAL EVALUATION IN A BALL}

Definition 4.1. Let $\mathbb{B}_{i}=\mathbb{B}_{h}\left(z_{i}\right), i=1,2, \cdots, n$, be open balls with radius $h$ centered at points $z_{i}=(x, y) \in \Omega$, such that $z_{i} \in G_{h}$ and $\overline{\mathbb{B}}_{i} \subset \bar{\Omega} \forall i=1,2, \cdots, n$. Moreover, for any index $i$ and $j$ with $i \neq j$ :

$$
\mathbb{B}_{i} \cap \mathbb{B}_{j} \neq \emptyset \Rightarrow z_{i} \in \partial \mathbb{B}_{j} \text { and } z_{j} \in \partial \mathbb{B}_{i} .
$$

Definition 4.1 provides a partial covering for $\bar{\Omega}$ denoted by $W$ such that $W=$ $\bigcup_{i} \mathbb{B}_{i}$, as shown in Fig. 2. We suppose that the function $\Psi$ defined on $\partial \Omega$ for problem $\Sigma$ can be extended to $\partial W$ (the boundary for $W$ ) in a suitable form, as it is usually done when a regular grid is introduced in a given domain. This extension can be made arbitrarily precise if one is prepared to decrease the step $h$ of $G_{h}$ (the radius $h$ of $\left.\mathbb{B}_{h}(\cdot)\right)$. With these considerations, we can replace the original problem $\Sigma$ by a similar boundary value problem involving the domain $W$ :

$$
\text { Problem }\left(\Sigma_{W}\right): \begin{cases}\Delta u(x, y)-\lambda^{2} u(x, y)=0, & (x, y) \in W, \\ u(x, y)=\Psi(x, y), & (x, y) \in \partial W .\end{cases}
$$

Now, let us depart from the original problem for a moment, and take advantage of the simple geometry of the Dirichlet problem defined in the interior of a ball. We first quote a result that establishes an analytical solution for the Dirichlet problem for the Helmholtz equation in a ball, and later in this section we announce and prove a result on the numerical evaluation for an integral on a circle, associated with the solution of the Helmholtz equation at a ball.

Lemma $4.1[7]$. Let $\mathbb{B}=\mathbb{B}_{h}(z)$ be a ball with finite radius $h$, centered at a point $z \in \Omega$, and let $\partial \mathbb{B}$ be the correspondent circle or the boundary for $\mathbb{B}$. Let $\Psi(x, y)$ be 


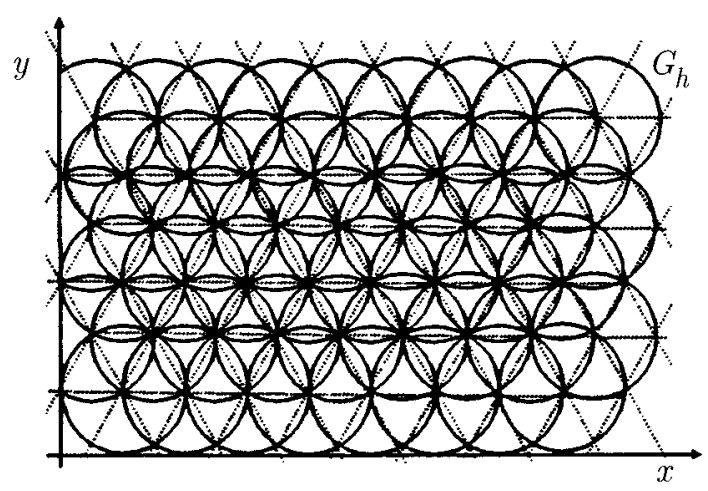

Figure 2. The regular covering $W$ for $\Omega$

such that on the circle $\partial \mathbb{B} \theta \mapsto \Psi(h, \theta) \in L_{2 \pi}^{p}$ for some $1 \leq p<\infty$, where $L_{2 \pi}^{p}$ is the class of $2 \pi$-periodic functions with the norm

$$
\left[\frac{1}{2 \pi} \int_{-\pi}^{+\pi}|f(\theta)|^{p} d \theta\right]^{\frac{1}{p}}
$$

Consider the following boundary value problem:

$$
\begin{cases}\Delta u(x, y)-\lambda^{2} u(x, y)=0, & (x, y) \in \mathbb{B} \\ u(x, y)=\Psi(x, y), & (x, y) \in \partial \mathbb{B} .\end{cases}
$$

The solution for (4.1) is given by:

$$
u(r, \theta)=\frac{a_{0}}{2} \frac{I_{0}(\lambda r)}{I_{0}(\lambda h)}+\sum_{n=1}^{\infty} \frac{I_{n}(\lambda r)}{I_{n}(\lambda h)}\left(a_{n} \cos n \theta+b_{n} \sin n \theta\right), \quad 0 \leq r<h,
$$

where $\left\{a_{n}\right\}, n=0,1,2, \ldots$, and $\left\{b_{n}\right\}, n=1,2, \ldots$, denote the Fourier coefficients of the function $\Psi$.

For our purposes, the interest is in the evaluation of $u$ at the center point $z$, thus $r=0$ in (4.2). Since $I_{n}(0)=0 \forall n \neq 0$, and $I_{0}(0)=1$, it follows that we can express

$$
u_{i, j}=\frac{1}{2 \pi I_{0}(\lambda h)} \int_{0}^{2 \pi} u(h, \theta) d \theta
$$

by applying the result in Lemma 4.1 to a ball $\mathbb{B}_{h}(z)$ with center at a point $z=$ $\left(x_{i}, y_{j}\right)$ belonging to the grid $G_{h}$. Thus, the grid function $u_{i, j}$ can be evaluated at point $z$, provided that the solution for $(\Sigma)$ on the circle $\partial \mathbb{B}$ is know. Recognizing that the value of $u(x, y)$ is only known in some (six) points on the circle $\partial \mathbb{B}$, we ought to use these point values for solving the integral in (4.3) numerically. It is possible to make an evaluation for the integral in (4.3) using the trapezoidal method for points regularly spaced as obtained with $G_{h}$. It attains great precision thanks to the fact that $\theta \mapsto u(h, \theta)$ is a periodical function [3]. The expression in (4.3) can be seen as an extension of the mean value theorem for the Helmholtz equation [2, chap. VII]. 
Theorem 4.1. Let $u$ be a function of class $\mathbb{C}^{6}$ in a neighborhood of the circle $\partial \mathbb{B}_{h}(z)$. The following evaluation holds.

$$
\left|\frac{1}{2 \pi} \int_{0}^{2 \pi} u(h, \theta) d \theta-\frac{1}{6} \sum_{k=1}^{6} u\left(h, \frac{k \pi}{3}\right)\right| \leq \frac{h^{6} D_{6}}{6 !}+O\left(h^{7}\right) .
$$

Proof. For the evaluation in (4.4), we pick up a point $z=\left(x_{i}, y_{i}\right) \in \Omega$, that may or may not belong to $G_{h}$. Let us denote by $\left(x_{i \pm 1}, y_{j \pm 1}\right):=\left(x_{i} \pm h / 2, y_{j} \pm h \sqrt{3} / 2\right)$ and $\left(x_{i \pm 2}, y_{j}\right):=\left(x_{i} \pm h, y_{j}\right)$ six equally spaced neighboring points at a distance $h$ from the point $\left(x_{i}, y_{j}\right)$. We also denote by $u_{z}$ and $u_{i \pm 1, j \pm 1}$ or $u_{i \pm 2, j}$ the value of function $u$ at point $z$ and at the six neighboring points, respectively, and we use the same notation for the derivatives. Expanding the representation for $u(x, y)$ by using the first $(n-1)$ th derivatives, it follows that

$$
\begin{gathered}
u\left(x_{i}+\alpha, y_{j}+\beta\right)=u_{z}+\alpha \frac{\partial u_{z}}{\partial x_{i}}+\beta \frac{\partial u_{z}}{\partial y_{i}}+\frac{1}{2 !}\left(\alpha^{2} \frac{\partial^{2} u_{z}}{\partial x_{i}^{2}}+2 \alpha \beta \frac{\partial^{2} u_{z}}{\partial x_{i} \partial y_{j}}+\beta^{2} \frac{\partial^{2} u_{z}}{\partial y_{j}^{2}}\right)+\cdots \\
+\frac{1}{n-1 !}\left\{\alpha^{n-1} \frac{\partial^{n-1} u_{z}}{\partial x_{i}^{n-1}}+\left(\begin{array}{c}
n-1 \\
1
\end{array}\right) \alpha^{n-2} \beta \frac{\partial^{n-1} u_{z}}{\partial x_{i}^{n-2} \partial y_{j}}+\cdots\right. \\
\left.+\left(\begin{array}{c}
n-1 \\
n-2
\end{array}\right) \alpha \beta^{n-2} \frac{\partial^{n-1} u_{z}}{\partial x_{i} \partial y_{j}^{n-2}}+\beta^{n-1} \frac{\partial^{n-1} u_{z}}{\partial y_{i}^{n-1}}\right\}+R_{n}
\end{gathered}
$$

where $R_{n}$ stands for the remainder of this representation. Denoting

$$
\begin{aligned}
\left(\alpha \frac{\partial}{\partial x_{i}}+\beta \frac{\partial}{\partial y_{i}}\right)^{n} u_{z}=\frac{1}{n !}\left\{\alpha^{n} \frac{\partial^{n} u_{z}}{\partial x_{i}^{n}}+\right. & \left(\begin{array}{c}
n \\
1
\end{array}\right) \alpha^{n-1} \beta \frac{\partial^{n-1} u_{z}}{\partial x_{i}^{n-1} \partial y_{j}}+\cdots \\
& +\left(\begin{array}{c}
n \\
n-1
\end{array}\right) \alpha \beta^{n-1} \frac{\partial^{n-1} u_{z}}{\partial x_{i} \partial y_{j}^{n-1}}+\beta^{n} \frac{\partial^{n} u_{z}}{\partial y_{i}^{n}}
\end{aligned}
$$

we can write equivalently

$$
u\left(x_{i}+\alpha, y_{j}+\beta\right)=u_{z}+\sum_{k=1}^{n-1} \frac{1}{k !}\left(\alpha \frac{\partial}{\partial x}+\beta \frac{\partial}{\partial y}\right)^{k} u_{z}+R_{n} .
$$

In the present situation $n=7$ and $R_{7} \approx O\left(h^{7}\right)$, and adopting $\alpha= \pm h$ and $\beta=0$, or $\alpha= \pm h / 2$ and $\beta= \pm h \sqrt{3} / 2$, it follows that $\left(x_{i}+\alpha, y_{j}+\beta\right)$ represents the six equally spaced neighboring points of $\left(x_{i}, y_{j}\right)$ at a distance $h$. For sake of notational simplicity, let $\sum u_{b}$ denote the sum of $u$ at the six neighboring points to point $z$. We have with a proper reference for the angle parameter $\theta$ that

$$
\sum u_{b}=\sum_{k=1}^{6} u\left(h, \frac{k \pi}{3}\right)
$$

and from (4.5)

$$
\sum u_{b}=6 u_{z}+\left.\frac{3 h^{2}}{2 !} \Delta u\right|_{z}+\left.\frac{3 h^{4}}{32} \Delta^{2} u\right|_{z}+\tau\left\{u_{z}\right\}
$$

The truncation error $\tau\left\{u_{z}\right\}$ can be found in [10] to be

$$
\tau\left\{u_{z}\right\}=\frac{h^{6}}{16 \times 6 !}\left(33 \frac{\partial^{6} u_{z}}{\partial x_{i}^{6}}+45 \frac{\partial^{6} u_{z}}{\partial x_{i}^{4} \partial y_{j}^{2}}+135 \frac{\partial^{6} u_{z}}{\partial x_{i}^{2} \partial y_{j}^{4}}+27 \frac{\partial^{6} u}{\partial y_{j}^{6}}\right)+O\left(h^{7}\right) ;
$$


using the definition of $D_{6}$ in $(2.1)$, we can write

$$
\left|\tau\left\{u_{z}\right\}\right| \leq \frac{15 h^{6}}{6 !} D_{6}+O\left(h^{7}\right)
$$

Now, we concentrate on the numerical solution of (4.3). Employing the trapezoidal method for solving the integral, one can write

$$
\frac{1}{2 \pi} \int_{0}^{2 \pi} u(h, \theta) d \theta=\frac{1}{6} \sum_{k=1}^{6} u\left(h, \frac{k \pi}{3}\right)+\tau_{t}\{u\},
$$

where $\tau_{t}\{u\}$ stands for the approximation error incurred by the trapezoidal method. We should also employ (4.6) to write (4.3) as:

$$
6 I_{0}(\lambda h) u_{z}=\sum u_{b}+6 \tau_{t}\{u\}
$$

Now, using the expression for $\sum u_{b}$ in (4.7) and substituting in the relation above, we have that

$$
\begin{aligned}
6 \tau_{t}\{u\}= & 6 I_{0}(\lambda h) u_{z}-6\left(u_{z}+\frac{h^{2} \Delta u}{4}+\frac{h^{4} \Delta^{2} u}{64}\right) \\
& -\frac{h^{6}}{16 \times 6 !}\left(33 \frac{\partial^{6} u_{z}}{\partial x_{i}^{6}}+45 \frac{\partial^{6} u_{z}}{\partial x_{i}^{4} \partial y_{j}^{2}}+135 \frac{\partial^{6} u_{z}}{\partial x_{i}^{2} \partial y_{j}^{4}}+27 \frac{\partial^{6} u}{\partial y_{j}^{6}}\right)+O\left(h^{7}\right) .
\end{aligned}
$$

One can use the fact that $\Delta u=\lambda^{2} u$ and $\Delta^{2} u=\lambda^{4} u$ and the series representation for $I_{0}(\lambda h)$ to write

$$
\begin{aligned}
6 \tau_{t}\{u\}= & \frac{6 h^{6} \Delta^{3} u}{2^{6}(3 !)^{2}}-\frac{h^{6}}{16 \times 6 !}\left(33 \frac{\partial^{6} u_{z}}{\partial x_{i}^{6}}+45 \frac{\partial^{6} u_{z}}{\partial x_{i}^{4} \partial y_{j}^{2}}+135 \frac{\partial^{6} u_{z}}{\partial x_{i}^{2} \partial y_{j}^{4}}+27 \frac{\partial^{6} u}{\partial y_{j}^{6}}\right) \\
& +O\left(h^{7}\right)
\end{aligned}
$$

also, introducing the form of $\Delta^{3} u$, we finally have that

$$
6 \tau_{t}\{u\}=\frac{3 h^{6}}{16 \times 6 !}\left(-\frac{\partial^{6} u_{z}}{\partial x_{i}^{6}}-15 \frac{\partial^{6} u_{z}}{\partial x_{i}^{4} \partial y_{j}^{2}}+15 \frac{\partial^{6} u_{z}}{\partial x_{i}^{2} \partial y_{j}^{4}}+\frac{\partial^{6} u_{z}}{\partial y_{j}^{6}}\right)+O\left(h^{7}\right)
$$

using the notation in $(2.1)$,

$$
\left|\tau_{t}\{u\}\right| \leq \frac{h^{6} D_{6}}{6 !}+O\left(h^{7}\right)
$$

and the result is therefore proven.

\section{Proof of Theorem 3.1 And Corollary}

Let us consider the problem $\Sigma$ or, alternatively, $\Sigma_{W}$ with no loss, as mentioned in section 4 . For the proof we now pick up a point $z=\left(x_{i}, y_{j}\right) \in \Omega$ that belongs to $G_{h}$ and is an interior point with respect to $\partial W$, and consider a ball $\mathbb{B}_{h}(z)$. We denote by $\left(x_{i \pm 1}, y_{j \pm 1}\right):=\left(x_{i} \pm h / 2, y_{j} \pm h \sqrt{3} / 2\right)$ and $\left(x_{i \pm 2}, y_{j}\right):=\left(x_{i} \pm h, y_{j}\right)$ the six points on the circle $\partial \mathbb{B}_{h}(z)$ that are also points of $G_{h}$ at a distance $h$ from the point $\left(x_{i}, y_{j}\right)$. We also denote by $u_{z}, u_{i \pm 2, j}$ and $u_{i \pm 1, j \pm 1}$ the value of function $u$ at point $z$ and at the six neighboring points, respectively, and we use the same 
notation for the derivatives. Using polar coordinates with an appropriate reference for the angular variable, we can also identify

$$
u\left(h, \frac{k \pi}{3}\right) \quad \text { for } k=1,2,3,4,5,6 .
$$

with each one of the neighboring points $\left(x_{i \pm 1}, y_{j \pm 1}\right)$ and $\left(x_{i \pm 2}, y_{j}\right)$. Using (4.3) and the trapezoidal rule for the numerical evaluation of the integral, we can write

$$
I_{0}(\lambda h) u_{i, j}=\frac{1}{2 \pi} \int_{0}^{2 \pi} u(h, \theta) d \theta=\frac{1}{6} \sum_{k=1}^{6} u\left(h, \frac{k \pi}{2}\right)+\tau_{t}\{u\},
$$

where, as in (4.9), $\tau_{t}\{u\}$ stands for the approximation error incurred by the trapezoidal rule, with an error evaluation given by Theorem 4.1. Thus,

$$
\left|6 I_{0}(\lambda h) u_{i, j}-\sum_{k=1}^{6} u\left(h, \frac{k \pi}{3}\right)\right| \leq\left|6 \tau_{t}\{u\}\right| \leq \frac{6 h^{6} D_{6}}{6 !}+O\left(h^{7}\right) .
$$

Let us denote by $U_{i, j}$ the grid function defined on $G_{h}$ that is the approximation for function $u_{i, j}$ obtained by the proposed method. It is such that $U_{i, j}=u_{i, j}$ at each point of the boundary $\partial W$ and at interior points $U_{i, j}$ satisfies the equation in Theorem 3.1, namely

$$
6 I_{0}(\lambda h) U_{i, j}=U_{i-2, j}+U_{i+2, j}+U_{i+1, j-1}+U_{i+1, j+1}+U_{i-1, j-1}+U_{i-1, j+1} .
$$

Similarly, using (5.1) and (5.2), we can write a difference equation for the grid function $u_{i, j}$ as follows:

$$
\begin{aligned}
6 I_{0}(\lambda h) u_{i, j}=u_{i-2, j}+u_{i+2, j} & +u_{i+1, j-1} \\
& +u_{i+1, j+1}+u_{i-1, j-1}+u_{i-1, j+1}+6 \tau_{t}\{u\} .
\end{aligned}
$$

Following this definition, we denote by $u$ and $U$ the vectors of appropriate dimension, each of them containing the values $u_{i, j}$ and $U_{i, j}$ respectively, at each point of $G_{h}$ whenever $\left(x_{i}, y_{j}\right)$ is also an interior point of $W$. Suppose that one can find $N_{0} \cdot M_{0}$ interior points in $G_{h}$, or equivalently, the same number of balls centered in the interior points of $W$ in $G_{h}$. We write for a proper ordering of these points:

$$
\begin{aligned}
u & :=\left[u_{1,1}, \cdots, u_{N_{0}, 1} ; u_{1,2}, \cdots, u_{N_{0}, 2} ; \cdots ; u_{1, M_{0}}, \cdots, u_{N_{0}, M_{0}}\right]^{T}, \\
U & :=\left[U_{1,1}, \cdots, U_{N_{0}, 1} ; U_{1,2}, \cdots, U_{N_{0}, 2} ; \cdots ; U_{1, M_{0}}, \cdots, U_{N_{0}, M_{0}}\right]^{T} .
\end{aligned}
$$

Also, let us define the error vector made up of the corresponding terms in (5.5) as:

$$
\tilde{\tau}_{t}\{u\}:=\left[\tau_{1,1}, \cdots, \tau_{N_{0}, 1} ; \tau_{1,2}, \cdots, \tau_{N_{0}, 2} ; \cdots ; \tau_{1, M_{0}}, \cdots, \tau_{N_{0}, M_{0}}\right]^{T} .
$$

From (5.4) and (5.5) we can form the following linear system of equations:

$$
\begin{gathered}
A u=b+6 \tilde{\tau}_{t}\{u\}, \\
A U=b,
\end{gathered}
$$

where $b$ is a vector formed by the elements $u_{i, j}$ associated to points at the boundary $\partial W$ whenever they are involved in the right-hand side of (5.4) and (5.5). Otherwise, when no boundary point is involved, the correspondent element of $b$ is null. 
When the domain is sufficiently regular, the matrix $A$ will have the following structure, after a proper definition and ordering:

$$
A=\left[\begin{array}{cccccc}
B_{1} & -J_{N_{0}} & 0 & 0 & \cdots & 0 \\
-J_{N_{0}}^{T} & B_{2} & -J_{N_{0}}^{T} & 0 & \cdots & 0 \\
0 & -J_{N_{0}} & B_{3} & -J_{N_{0}} & \cdots & 0 \\
\vdots & & \vdots & \ddots & & \vdots \\
0 & \cdots & 0 & -J_{N_{0}}^{T} & B_{M_{0}-1} & -J_{N_{0}}^{T} \\
0 & \cdots & 0 & 0 & -J_{N_{0}} & B_{M_{0}}
\end{array}\right]
$$

with dimension $N_{0} M_{0} \times N_{0} M_{0}$. Matrices $B_{i}$ are defined by:

$$
B_{i}=6 I_{0}(\lambda h) \mathbb{I}_{N_{0}}-C_{N_{0}} \quad \text { for } i=1,2, \cdots, M_{0},
$$

where $\mathbb{I}_{N_{0}}$ is the identity matrix with dimension $N_{0} \times N_{0}$. The matrices $J_{N_{0}}$ and $C_{N_{0}}$ are defined by

$$
J_{N_{0}}=\left[\begin{array}{ccccc}
1 & 1 & 0 & \cdots & 0 \\
0 & 1 & 1 & \cdots & 0 \\
\vdots & & \ddots & & \vdots \\
0 & \cdots & 0 & 1 & 1 \\
0 & \cdots & 0 & 0 & 1
\end{array}\right]_{N_{0} \times N_{0}}, C_{N_{0}}=\left[\begin{array}{ccccc}
0 & 1 & 0 & \cdots & 0 \\
1 & 0 & 1 & \cdots & 0 \\
\vdots & & \ddots & & \vdots \\
0 & \cdots & 1 & 0 & 1 \\
0 & \cdots & 0 & 1 & 0
\end{array}\right]_{N_{0} \times N_{0}}
$$

Before the proof of Theorem 3.1 we pause to prove the result in Lemma 3.1.

Proof of Lemma 3.1. Notice first that the standard finite difference method applied to the same triangular mesh grid would provide similar matrices as in (5.8)-(5.10), with the exception that the sub-matrices $B_{i}$ would have the following form:

$$
B_{i}=6 T_{1}(\lambda h) \mathbb{I}_{N_{0}}-C_{N_{0}} \quad \text { for } i=1,2, \cdots, M_{0} .
$$

In addition, for a matrix $A=\left\{a_{i j}\right\}$ generated by any of the two methods, one can verify that

$$
a_{i j}=0 \text { or } a_{i j}=-1, \quad \text { for } i \neq j
$$

moreover,

$$
-6 \leq \sum_{j \neq i} a_{i j} \leq 0, \quad \text { for any } i=0,1, \ldots, N_{0} M_{0} .
$$

Notice that this structure of matrix $A_{I_{0}}$ or $A_{T_{1}}$ is invariant, even if the domain is not strictly rectangular or triangular. With $\Sigma=I_{0}$ or $\Sigma=T_{1}$, the norm definition yields

$$
\left\|A_{\Sigma} v\right\|_{\infty}=\sup _{i=1, \ldots, N_{0} M_{0}}\left\{\left|6 \Sigma(\lambda h) v_{i}-\sum_{j \neq i} a_{i j} v_{j}\right|\right\}
$$

and supposing that $\|v\|_{\infty}=v_{i_{0}}$ for some $i_{0}$, we evaluate

$$
\begin{aligned}
\left\|A_{\Sigma} v\right\|_{\infty} & \geq\left|6 \Sigma(\lambda h) v_{i_{0}}-\sum_{j \neq i_{0}} a_{i j} v_{j}\right| \\
& \geq 6(\Sigma(\lambda h)-1) v_{i_{0}}
\end{aligned}
$$


Similarly, let us suppose that $\|v\|_{\infty}=-v_{i_{0}}$ for some $i_{0}$, and evaluate

$$
\begin{aligned}
\left\|A_{\Sigma} v\right\|_{\infty} & \geq-6 \Sigma(\lambda h) v_{i_{0}}+\sum_{j \neq i_{0}} a_{i j} v_{j} \\
& \geq-6(\Sigma(\lambda h)-1) v_{i_{0}} ;
\end{aligned}
$$

from (5.11) and (5.12) the result in Lemma 3.1 is proven.

Proof of Theorem 3.1. The proof of Theorem 3.1 now follows in a straightforward manner by subtracting (5.7) from (5.6):

$$
A(u-U)=6 \tilde{\tau}_{t}\{u\}
$$

and taking the identity

$$
\|A(u-U)\|_{\infty}=6\left\|\tilde{\tau}_{t}\{u\}\right\|_{\infty} .
$$

The result follows from Lemma 3.1.

Proof of Corollary 3.1. The proof is immediate from Theorem 3.1, since it is enough to verify that

$$
I_{0}(\lambda h)-1=\sum_{k=1}^{\infty} \frac{(\lambda h)^{2 k}}{2^{2 k}(k !)^{2}}=O\left(h^{2}\right)
$$

thus, in view of (3.2) it follows that

$$
\|u-U\|_{\infty} \leq \frac{1}{O\left(h^{2}\right)}\left\|\tau_{t}\{u\}\right\|_{\infty}=O\left(h^{4}\right)
$$

proving the result.

\section{NUMERICAL EXPERIMENTS}

The examples in this section are drawn from convection-diffusion problems of the form $\Delta u+\alpha \cdot \nabla u=0$. They are transformed to the standard Helmholtz form $\Delta w-\lambda^{2} w=0$ with the simple transformation

$$
w=u \exp \left(\frac{1}{2} \alpha \cdot p\right), \text { with } p=(x, y)^{T} \text { and } \lambda^{2}=\frac{1}{4} \alpha \cdot \alpha^{T} .
$$

The comparisons however are presented in terms of the original function $u$.

Test Problem 6.1. Consider the boundary value problem with dominated convection term

$$
\begin{cases}-\varepsilon\left(\frac{\partial^{2} u}{\partial x^{2}}+\frac{\partial^{2} u}{\partial y^{2}}\right)+\frac{\partial u}{\partial x}=0, & 0 \leq x, \quad y \leq 1 \\ u(x, 0)=0, \quad u(x, 1)=0, & 0 \leq x \leq 1 \\ u(0, y)=\sin \pi y, \quad u(1, y)=2 \sin \pi y, & 0 \leq y \leq 1\end{cases}
$$

The Problem 6.1 was specially considered by Gartland in [4], in which a specific difference method for the five-point rectangular mesh grids called Discrete Weighted Mean Approximation (DWMA), was proposed involving the modified Bessel function. This problem is also studied by Gupta et al. in [8] using the SCHOS method, with advantage to the former method. Using the variable transformation in (6.1), 
the equation (6.2) is posed in the Helmholtz canonical form; the exact solution of (6.2) is given by

$$
u(x, y)=\frac{e^{\lambda x}\left[2 e^{-\lambda} \sinh \sigma x+\sinh \sigma(1-x)\right] \sin \pi y}{\sinh \sigma}, \quad \sigma^{2}=\pi^{2}+\lambda^{2},
$$

with $\lambda=1 / 2 \varepsilon$. For comparison purposes, we present in Table 1 the evaluations for the relative error obtained from the numerical experiments carried out for the FDS and the MVS on triangular mesh grids, together with the results using the SCHOS presented in [8]. Table 1 shows that the accuracy is clearly in favor of the MVS.

TABle 1. Test Problem 1.

Maximum relative error $h=1 / 32$

\begin{tabular}{rlcc}
\hline$\lambda$ & \multicolumn{1}{c}{ FDS } & SCHO $[8]$ & MVS \\
\hline 5 & $0.1028(-5)^{*}$ & $0.6011(-4)$ & $0.8634(-6)$ \\
10 & $0.2651(-4)$ & $0.1399(-3)$ & $0.7184(-5)$ \\
20 & $0.9842(-3)$ & $0.1511(-2)$ & $0.1283(-3)$ \\
50 & 0.1040 & $0.3517(-1)$ & $0.9542(-2)$ \\
\hline$*_{0.1028(-5)=0.1028 \times 10^{-5}}$
\end{tabular}

The maximum absolute error for different values of mesh grid size $h$ for the MVS is presented in Table 2, with numerical estimates for the precision error order, by considering two successive error evaluations.

TABle 2. Test Problem 1 with MVS.

Maximum absolute error and

the estimated error order

\begin{tabular}{rlclcc}
\hline$\lambda$ & \multicolumn{1}{c}{$h=1 / 8$} & Order & \multicolumn{1}{c}{$h=1 / 16$} & Order & $h=1 / 32$ \\
\hline 5 & $0.2961(-3)$ & 3.85 & $0.2055(-4)$ & 3.57 & $0.1725(-5)$ \\
10 & $0.2692(-2)$ & 3.76 & $0.1983(-3)$ & 3.79 & $0.1436(-4)$ \\
20 & $0.3922(-1)$ & 3.46 & $0.3557(-2)$ & 3.79 & $0.2563(-3)$ \\
50 & 1.1056 & 2.51 & 0.1945 & 3.35 & $0.1907(-1)$ \\
\hline
\end{tabular}

We observed from the experiments with the FDS and the MVS that when $\lambda h \simeq 2$ $(\lambda=30, h=1 / 16)$ the FDS yields poor results with relative errors larger than $10 \%$, whereas the MVS presents an error of $1.1 \%$. The error level about $10 \%(9.8 \%)$ is only attained by the MVS when $\lambda=50$ and $h=1 / 16$. When the product $\lambda h>2$ is adopted $(\lambda=40, h=1 / 16$ or $\lambda=75, h=1 / 32)$ the error increases to values as large as $49 \%$, indicating that the FDS method is incapable of handling problems when $\lambda h>2$, whereas the MVS yields an error of $3.8 \%$ and $6.11 \%$ for $\lambda=40$, $h=1 / 16$ and $\lambda=75, h=1 / 32$, respectively. Thus, trying with larger values for the product $\lambda h$ with the MVS, numerical experiments show that large errors will only appear when $\lambda h>4(\lambda=64, h=1 / 16$ or $\lambda=128, h=1 / 32)$, with relative error values as large as $27 \%$. 
In Table 3, a comparison among the FDS, SCHOS, DWMA and the MVS is presented by means of the maximum absolute error and numerical estimates for the precision error order, using successive error evaluations. When $\lambda=50$ the FDS method yields large errors; we omit the results and use instead the results for the maximum absolute error presented in [9] for the DWMA and SCHOS for comparison purposes.

TABLE 3. Test Problem 6.1

Compared maximum absolute

error and estimated error order

\begin{tabular}{rrlcllll}
\hline$\lambda$ & $h^{-1}$ & \multicolumn{1}{c}{ FDS } & Order & SCHOS[8] & Order & MVS & \\
\hline 20 & 8 & 0.3289 & & 0.1256 & & $0.3922(-1)$ & \\
& 16 & $0.2739(-1)$ & 3.58 & $0.2009(-1)$ & 2.65 & $0.3557(-2)$ & 3.46 \\
& 32 & $0.1967(-2)$ & 3.79 & $0.1712(-2)$ & 3.55 & $0.2563(-3)$ & 3.79 \\
\hline & & & & & & & \\
\hline$\lambda$ & $h^{-1}$ & DWMA[9] & Order & SCHOS[9] & Order & MVS & Order \\
\hline 50 & 8 & $0.3685(-1)$ & & 0.4249 & & 1.1056 & \\
& 16 & $0.5812(-1)$ & - & 0.1670 & 1.35 & 0.1945 & 2.51 \\
& 32 & $0.4993(-1)$ & 0.22 & $0.3365(-1)$ & 2.31 & $0.1907(-1)$ & 3.35 \\
\hline
\end{tabular}

One may observe from Table 3 that the solution with the DWMA is more precise than that yielded by the MVS when the mesh is crude. When the grid is refined the solution with the MVS shows a fast convergence of $O\left(h^{3.35}\right)$.

Test Problem 6.2 (Borrowed from [8] and [13]). Consider the boundary value problem

$$
\begin{cases}\frac{\partial^{2} u}{\partial x^{2}}+\frac{\partial^{2} u}{\partial y^{2}}=P \cos \theta \frac{\partial u}{\partial x}+P \sin \theta \frac{\partial u}{\partial y}, & 0 \leq x, y \leq 1 \\ u(x, 0)=0, \quad u(x, 1)=0, & 0 \leq x \leq 1 \\ u(0, y)=4 y(1-y), \quad u(1, y)=0, & 0 \leq y \leq 1\end{cases}
$$

Problem 6.2 represents the convection of temperature or concentration in a fluid moving with a uniform velocity at an angle $\theta$ with respect to the $x$-axis. Using the variable transformation in (6.1) with $\alpha=(-P \cos \theta-P \sin \theta)^{T}$, equation (6.3) is posed in the Helmholtz canonical form. The exact solution of (6.3) is given by [8]

$$
u(x, y)=e^{P(x \cos \theta+y \sin \theta) / 2} \sum_{n=1}^{\infty} A_{n} \sinh \left[\sigma_{n}(1-x)\right] \sin (n \pi y)
$$

where

$$
A_{n}=\frac{8}{\sinh \sigma_{n}} \int_{0}^{1} t(1-t) e^{-(P \sin \theta) t} \sin (n \pi t) d t, \quad \sigma_{n}^{2}=n^{2} \pi^{2}+P^{2} / 4 .
$$


In Table 4 we transcribe the maximum absolute error presented in [8] for the SCHOS method and compare them with the numerical results obtained with the FDS and the MVS method for $P=40, h=1 / 8,1 / 16,1 / 32$ and $\theta=0^{\circ}, \pi / 8, \pi / 4$. Notice the consistent good behavior of the MVS method, presenting an error that is 3.7 smaller in its minimum and 17 times smaller in its best comparison with the FDS method; the same comparison with the SCHOS method ranges from 1.6 to 7.2 times smaller. Notice that the performance of the MVS is not affected by the variation of $\theta$, as it may occur with other methods, see [13].

TABle 4. Test Problem 2.

Compared maximum absolute error

and estimated error order $(P=40)$

\begin{tabular}{rrllllcl}
\hline \multicolumn{1}{r}{$\theta$} & $h^{-1}$ & \multicolumn{1}{c}{ FDS } & Order & SCHOS[8] & Order & MVS & Order \\
\hline $0^{\circ}$ & 8 & 0.3356 & & $0.8280(-1)$ & & $0.3912(-1)$ & \\
& 16 & $0.2796(-1)$ & 3.58 & $0.1323(-1)$ & 2.65 & $0.3554(-2)$ & 3.46 \\
& 32 & $0.2003(-2)$ & 3.80 & $0.1123(-2)$ & 3.55 & $0.2549(-3)$ & 3.80 \\
\hline$\pi / 8$ & 8 & 0.2993 & & $0.6931(-1)$ & & $0.1770(-1)$ & \\
& 16 & $0.2048(-1)$ & 3.87 & $0.1019(-1)$ & 2.77 & $0.1630(-2)$ & 3.44 \\
& 32 & $0.1546(-2)$ & 3.72 & $0.8128(-3)$ & 3.64 & $0.1124(-3)$ & 3.86 \\
\hline$\pi / 4$ & 84 & 0.1214 & & $0.4932(-1)$ & & $0.2321(-1)$ & \\
& 16 & $0.1110(-1)$ & 3.45 & $0.5977(-2)$ & 3.04 & $0.2889(-2)$ & 3.00 \\
& 32 & $0.9515(-3)$ & 3.54 & $0.4066(-3)$ & 3.88 & $0.2551(-3)$ & 3.50 \\
\hline
\end{tabular}

In a final comparison, we employ the numerical results obtained with the FDS and the MVS for the test Problem 6.2 with $P=80$ and $\theta=\pi / 8$. In Fig. 3 (a) the exact solution is presented, in Fig. 3 (b) the solution obtained by the MVS is pictured, in Figs. 3 (c) and 3 (d) the error values for the MVS and the FDS are displayed, respectively. The maximum absolute error in the FDS is $0.5362(-1)$ and for the MVS it reaches 0.3759(-2), thus more than ten times smaller than the error yielded by the former. The same problem was also solved in [13] using a 9-point scheme called QIS. For the same values of $P$ and $h$, the QIS seems to be more precise when the grid is crude, although both methods MVS and QIS yield comparable error values when the grid is refined. 


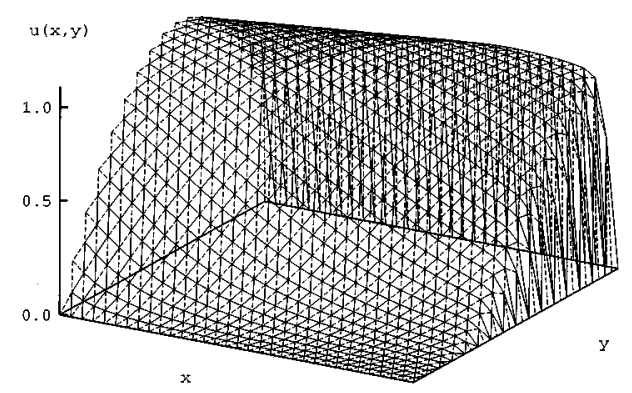

(a)

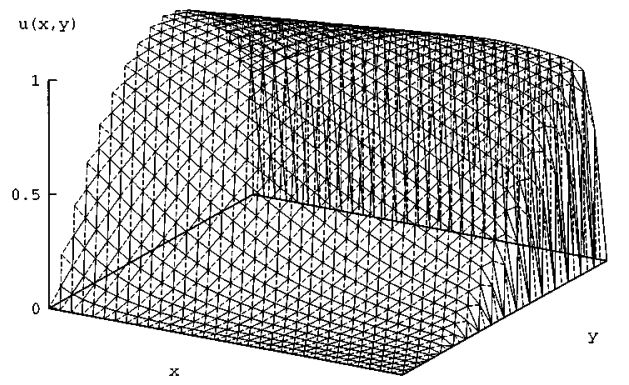

(b)

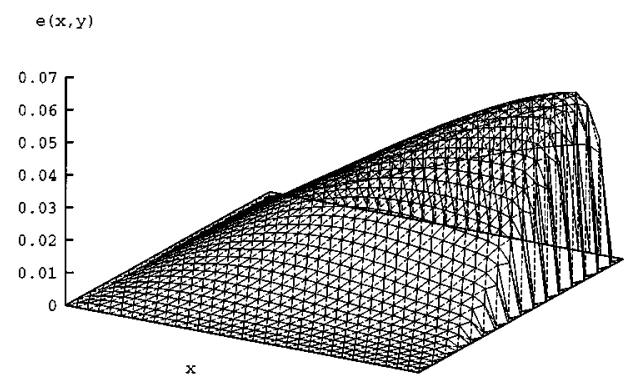

(c)

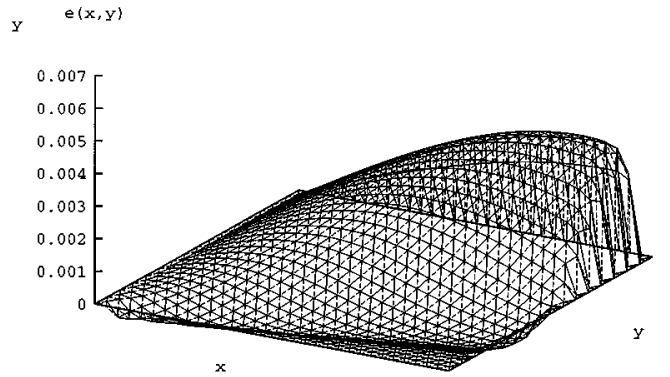

(d)

Figure 3. Numerical solutions for test Problem 6.2: (a) - Exact solution; (b) - MVS solution; (c) - Error yielded by the MVS; (d) - Error yielded by the FDS 


\section{Concluding Remarks}

In each of the numerical examples that were implemented by the authors, the MVS provides solutions that are consistently better than those obtained by other methods with respect to the maximum relative and maximum absolute error, including methods with stencils involving more than 7 points. The exception to this rule was only encountered in the comparison with the QIS method for some parameter values in the test Problem 6.2. It is important to mention that the comparison with the FDS has always yielded better results in the examples than those anticipated by the analysis in section 3 , in the comparison of the ratio between the error upper bounds for both methods, as pictured in Fig. 1.

\section{REFERENCES}

1. G. Birkhoff and S. Gulati, Optimal few-point discretizations of linear source problems, SIAM J. Numer. Anal. 11 (4) (1974), 700-728. MR 50:15371

2. R. Courant and D. Hilbert, Methods of mathematical physics (partial differential equations), vol. II, John Wiley \& Sons, New York, 1962. MR 25:4216

3. P. J. Davis and P. Rabinowitz, Methods of numerical integration, Academic Press, New York, 1975. MR 56:7119

4. E. C. Gartland Jr., Discrete weighted mean approximation of model convection-diffusion equation, SIAM J. Sci. Stat. Comp. 3 (4) (1982), 460-472. MR 84j:65058

5. D. Gilbarg and N. S. Trudinger, Elliptic partial differential equations of second order, Springer-Verlag, New York, 1983. MR 86c:35035

6. G. H. Golub and C. F. V. Loan, Matrix computations, The John Hopkins Univ. Press, Baltimore, 1984.

7. J. Górowski, On some properties of the solution of the Dirichlet problem for the Helmholtz equation in the interior and exterior of a circle, Demonstratio Math. 19 (2) (1986), 303-315. MR 88j:35040

8. M. M. Gupta, R. P. Manohar and J. W. Stephenson, A single cell high order scheme for the convection-diffusion equation with variable coefficients, Int. J. Numer. Methods Fluids 4 (1984), 641-651. MR 85f: 76010

9. M. M. Gupta, R. P. Manohar and J. W. Stephenson, High-order difference schemes for twodimensional elliptic equation, Numer. Methods for Partial Diff. Equations 1 (1985), 71-80. MR 87m:65150

10. L. V. Kantorovich and V. I. Krylov, Approximate methods of higher analysis, Intersc. Publish. Inc., New York, 1958. MR 21:5268

11. R. P. Manohar and J. W. Stephenson, Single cell high order difference methods for the Helmholtz equation, J. Comput. Phys. 51 (1983), 444-453.

12. T. P. Mathew, Schwarz alternating and iterative refinement methods for mixed formulations of elliptic problems, part I and part II: algorithms and numerical results, Numer. Math. 65 (4) (1993), 445-492. MR 94m:65171; MR 94m:65172

13. G. D. Stubley, G. D. Raithby and A. B. Strong, Proposal for a new discrete method based on an assessment of discretization errors, Num. Heat Transfer 3 (1980), 411-428.

14. J. B. R. do Val and M. G. Andrade Fo., On the numerical solution of the Dirichlet problem for Helmholtz equation, Applied Math. Letters 9 (1996), 85-89.

Depto. de Ciencias de Computacao e Estatistica, Instituto de Ciencias Matematica de Sao Carlos, Universidade de Sao Paulo, C.P. 668 - Sao Carlos - SP, 13.560-970 - Brasil

E-mail address: Marinho@icmsc.usp.br

Depto. de Telemática, Fac. de Eng. Elétrica, Universidade Estadual de Campinas UNICAMP, C.P. 6101, 13081-970 - CAMPINAS - SP, BRASIL

E-mail address: jbosco@dt.fee.unicamp.br 